\title{
Metallic Plasmonic Nano-antenna for Wireless Optical Communication in Intra-body Nanonetworks
}

\author{
Mona Nafari \\ Department of Electrical Engineering \\ University at Buffalo, \\ The State University of New York \\ Buffalo, NY, USA \\ monanafa@buffalo.edu
}

\author{
Josep Miquel Jornet \\ Department of Electrical Engineering \\ University at Buffalo, \\ The State University of New York \\ Buffalo, NY, USA \\ jmjornet@buffalo.edu
}

\begin{abstract}
Nanonetworks consist of nano-sized communicating devices which are able to perform simple tasks at the nanoscale. Nanonetworks are the enabling technology for unique applications, including intra-body health-monitoring and drug delivery systems. In this paper, metallic plasmonic nanoantennas for wireless optical communication in intra-body nanonetworks are modeled and analyzed. More specifically, a unified mathematical framework is developed to investigate the performance in reception of gold-based nano-dipole antennas. This framework takes into account the metal properties, i.e., its dynamic complex conductivity and permittivity; the propagation properties of Surface Plasmon Polariton waves on the nano-antenna, i.e., their confinement factor and propagation length; the antenna geometry, i.e., length and radius, and the antenna fundamental resonance frequency, and it can be utilized to obtain the plasmonic currents on the nano-antenna generated by an incident EM filed. In addition to numerical results, the analytical models are validated by means of simulations with COMSOL Multiphysics. The developed framework will guide the design and development of novel nano-antennas suited for wireless optical communication in intra-body nanonetworks.
\end{abstract}

\section{Categories and Subject Descriptors}

C.2.1 [Computer-Communication Networks]: Network Architecture and Design - Wireless Communication; H.1.1 [Models and Principles]: Systems and Information Theory-Information Theory

\section{General Terms}

Theory

\section{Keywords}

Nanophotonics, Plasmonics, Nano-antenna, Nanonetworks

\section{INTRODUCTION}

Nanotechnology is providing the engineering community with a new set of tools to design and manufacture novel nanoscale devices, which are able to perform simple tasks, such as computing, data storing, sensing and actuation. The integration of several of these nano-devices into a single entity will enable the development of advanced nanoscale machines. By means of communication, nanomachines will be able to organize themselves in networks, or nanonetworks, and complete more complex tasks in a distributed fashion [1]. For example, a collection of nanomachines distributed within the human body or traveling with the blood stream could serve as the basis of novel in-vivo health monitoring systems. Among others, nanomachines could be able to transmit their sensing information in a multi-hop fashion to a gateway or sink, or coordinate between them in case that a joint response to an event is needed.

There are several technologies that could potentially enable the communication between intra-body nanosensors. Among others, molecular communication, which is based on the exchange of molecules to transmit information, has been thoroughly investigated for several years [2]. This mechanism is naturally used by cells to exchange information and to coordinate their actions, and could be enabled by means of synthetic biology. However, the very low achievable data rates in molecular communications could drastically limit the usefulness of nanonetworks. Another very relevant technology is the utilization of ultrasonic communication, based on the utilization of very high frequency acoustic waves [20]. However, for the time being, the size and power limitations of ultrasonic acoustic transducers pose a challenge in their integration within cell-sized nanomachines.

From the electromagnetic (EM) perspective, the miniaturization of a conventional metallic antenna to meet the size requirements of a nanomachine would theoretically result in very high resonance frequencies. For example, according to classical antenna theory, a one-micrometer-long metallic antenna would radiate at approximately $150 \mathrm{THz}$. This is only true if the material of the antenna building components is assumed to be a Perfect Electrical Conductor (PEC), i.e., a material with infinite conductivity. However, at the aforementioned frequencies, metals no longer behave as PEC [23]. On the contrary, the conductivity of metals such as gold or silver [17]; metamaterials, i.e., engineered materials with rationally designed arrangements of nano-structured building blocks [5]; as well as nanomaterials such as graphene [21], is a frequency-dependent complex magnitude. 
The material conductivity affects the way in which a current wave propagates in the nano-antenna. In particular, the global oscillations of electrical charge in close proximity to the surface of the antenna building components, i.e., within the so called penetration depth, results into the excitation of a confined EM wave at the surface of the antenna. This peculiar EM wave is commonly referred to as a Surface Plasmon Polariton (SPP) wave. The frequency at which SPP waves are excited depends on the material conductivity of the antenna building components. For example, graphene supports the propagation of SPP waves at frequencies as low as in the THz band $(0.1-10 \mathrm{THz})[22]$, whereas in noble metals such as gold or silver, SPP waves are only observed at several tens of $\mathrm{THz}$ and above [23].

In this work, we are interested in designing plasmonic nano-antennas able to efficiently operate in-vivo, i.e., directly in contact or inside the human body. It is well-known that the propagation of EM waves inside the human body becomes more challenging as the frequency increases, mainly because of the absorption by biological tissues with a high content of water as well as fat, melatonin or hemoglobin, among others [12]. For example, intra-body communication at $\mathrm{THz}$-band frequencies enabled by graphene-based plasmonic nano-antennas [15] would suffer from very high absorption from water molecules [14].

For this, we advocate for the use of the near-infrared (NIR) or optical window for communication in intra-body nanonetworks. This is the frequency range between $225 \mathrm{THz}$ and $460 \mathrm{THz}$ (or, equivalently, from $650 \mathrm{~nm}$ to $1350 \mathrm{~nm}$ in terms of wavelength). In this window, the absorption of EM radiation by biological tissues is very small, in the order of $0.01 \mathrm{~m}^{-1}$ [12], or four orders of magnitude lower than the absorption at THz-band frequencies [19]. Moreover, plasmonic nano-devices at optical frequencies have already been utilized for in-vivo applications. For example, many of the biological nanosensors developed to date exploit the behavior of plasmons in nanomaterials and nano-particles $[6,8]$.

Within this context, the field of optical nano-antennas has been gaining momentum. Among others, in [4, 18], the state of the art in plasmonic nano-antennas for optical frequencies based on noble metals is described in detail. In [7], the propagation properties of SPP waves on nanowires are analyzed by taking into account the radial boundary condition along the nano-antenna. Then in [11], the impact of the nano-antenna caps is also taken into account. More recently, in [9], the nano-antenna fields are characterized, starting from the estimated propagation constant of SPP waves on nanowires obtained by means of experimental measurements. Despite all these results, a unified framework able to relate the material properties, the SPP wave behavior and the nano-antenna performance is missing.

In this paper, we model and analyze the performance of metallic plasmonic nano-antennas for intra-body optical communication. More specifically, we develop a unified mathematical framework to investigate the performance in reception of gold-based nano-dipole antennas. This framework relates the metal properties (conductivity and permittivity), the SPP wave propagation properties (confinement factor and propagation length), the antenna geometry (length and radius), and the antenna fundamental resonance frequency and can be utilized to obtain the plasmonic current in the nano-antenna generated by an incident EM filed. In addition to numerical results, we validate our analysis by

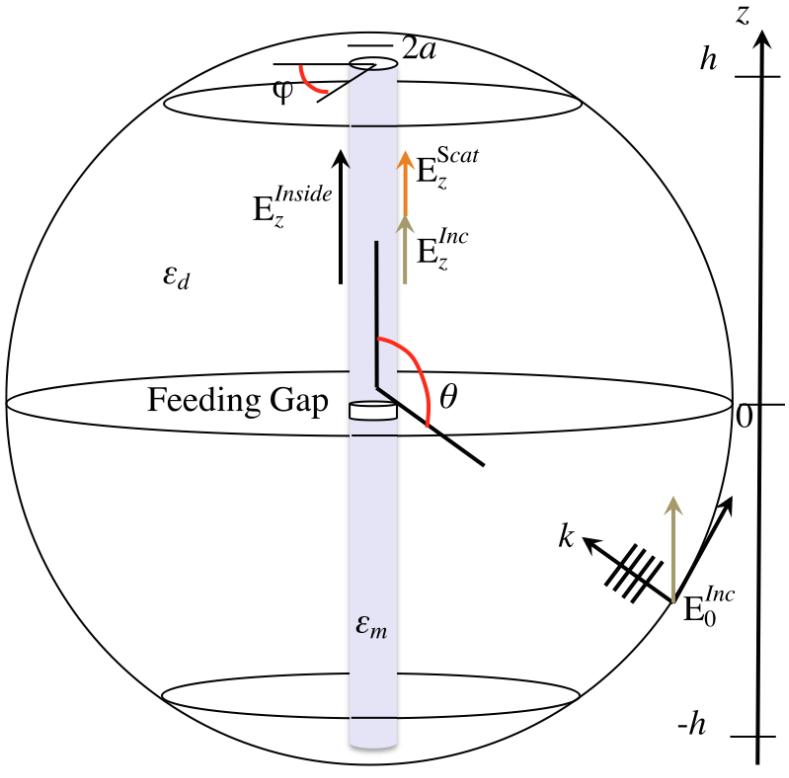

Figure 1: Plasmonic nano-dipole antenna considered in our analysis.

means of simulations with COMSOL Multi-physics.

The remainder of this paper is organized as follows. In Section 2, we review the fundamental working principle of a plasmonic nano-antenna, describe the conductivity model utilized in our analysis and analyze the SPP wave propagation properties. Then, in Section 3, we formulate the EM fields on and inside the antenna, and obtain a semiclosed form expression for the plasmonic current in the nanoantenna. The numerical and simulation results are presented in Section 4, and the paper is concluded in Section 5.

\section{PLASMONIC NANO-ANTENNA MODEL}

\subsection{Working Principle}

The plasmonic nano-antenna design considered in our analysis is shown in Figure 1. The nano-antenna consists of two identical metallic arms with a feeding/receiving gap in between. The working principle of the nano-antenna is as follows. Due to space constraints, we focus on its functioning in reception, but the same behavior holds in transmission due to the antenna reciprocity principle [3]. Then, in particular,

- Consider an incident plane wave with polarization parallel to the plane defined by the nano-antenna long axis and the direction of the wave propagation. Following the notation in Figure 1, in our analysis we are interested in the incident electric field component parallel to the $z$-axis, $E_{z}^{I n c}$, which is given by:

$E_{z}^{I n c}(r, z)=E_{0}^{I n c}(r) \sin \theta e^{j\left(k_{0} \cos \theta\right) z}=E_{\|}^{I n c}(r) e^{j k_{\| \mid} z}$,

where $E_{0}^{I n c}$ stands for the field strength, $k_{0}$ refers to the wave vector, and $\theta$ is the angle of incidence. When $E_{z}^{I n c}$ irradiates the nano-antenna, it excites the free electrons within the antenna penetration depth. The electronic response of the nano-antenna to an EM field 
is determined by the conductivity of its building material. In Section 2.2, we describe the conductivity model for metals that we utilize in our analysis.

- At the interface between the antenna metallic arms and the surrounding medium, longitudinal SPP waves that propagate along the $z$-axis are excited. The SPP wave vector, $k_{s p p}$ is mainly determined by the antenna geometry, namely, the antenna arm radius and length, as well as on the real and imaginary part of the material conductivity. In Section 2.3, we deduce the dispersion equation for SPP waves on the antenna and analyze their propagation properties as functions of the material conductivity and antenna geometry.

- To enhance the detection of the incident EM wave, we are interested in designing a plasmonic resonant cavity. In Section 3, we analyze the nano-antenna in reception, by starting from the formulation of the electric field incident to the antenna, scattered by the antenna and propagating inside the antenna.

\subsection{Optical Conductivity Model for Metals}

The conductivity of metals at optical frequencies is a complex magnitude. There are many analytical models for the conductivity of metals and, in our analysis, we utilize the well-established Drude model [13]. Accordingly, the metal complex conductivity, $\sigma$, or similarly, its complex permittivity, $\varepsilon_{m}$, can be written as functions of the angular frequency, $\omega=2 \pi f$, as follows:

$$
\begin{aligned}
\sigma(\omega) & =-j\left(\varepsilon_{m}(\omega)-\varepsilon_{\infty}\right) \varepsilon_{0} \omega \\
& =-j \omega \varepsilon_{0}\left[-\frac{\omega_{p}^{2} \tau_{D}^{2}}{1+\omega^{2} \tau_{D}^{2}}+j \frac{\omega_{p}^{2} \tau_{D}}{\omega\left(1+\omega^{2} \tau_{D}^{2}\right)}\right],
\end{aligned}
$$

where $\varepsilon_{0}$ is the vacuum permittivity, $\varepsilon_{\infty}$ is 1 for metals [16], $\omega_{p}$ stands for the plasma wave frequency, and $\tau_{D}$ is the electron relaxation time.

In this paper, we consider the use of gold $(\mathrm{Au})$ to build the nano-antennas [13] with values of $3.3 \cdot 10^{-14} \mathrm{~s}$ and 13.8 . $10^{15} \mathrm{rad} / \mathrm{s}$ for $\omega_{p}$ and $\tau_{D}$, respectively. In Figure 2, the real and imaginary parts of the relative permittivity for gold are shown as functions of frequency. The absolute value of real and imaginary part of permittivity in higher frequencies tends to decrease which considerably affects propagation of $\mathrm{SPP}$ waves, as we show in the next section.

\subsection{Surface Plasmon Polariton Wave Dispersion Equation}

In this section, we analytically study the propagation properties of SPP waves in the nano-antenna, by taking into account both its geometry and the complex conductivity of its building material. More specifically, we are interested in characterizing the complex SPP wave vector $k_{s p p}$. The real part of the wave vector,

$$
R e\left\{k_{s p p}\right\}=\frac{2 \pi}{\lambda_{s p p}},
$$

determines the SPP wavelength $\lambda_{s p p}$, as well as the confinement factor $k_{s p p} / k_{0}$. The imaginary part, $\operatorname{Im}\left\{k_{s p p}\right\}$, determines the SPP decay or, inversely,

$$
L=\frac{1}{2 \operatorname{Im}\left\{k_{s p p}\right\}},
$$

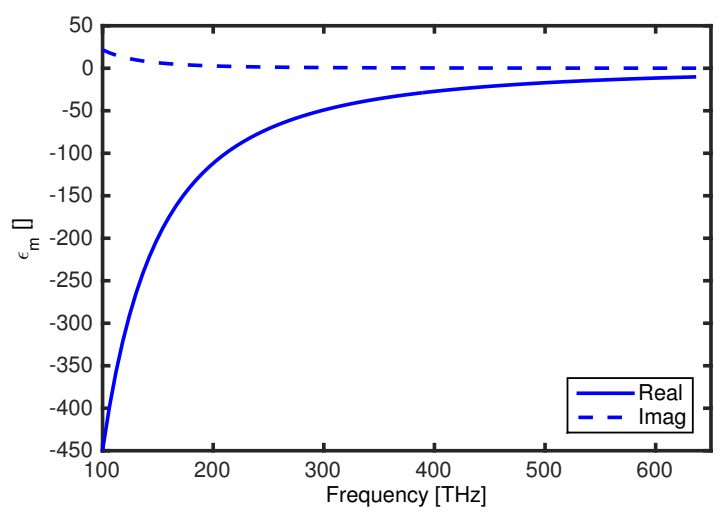

Figure 2: Real and imaginary parts of the relative permittivity for gold as functions of frequency.

determines the SPP propagation length, which is defined as the distance at which the SPP field strength has decreased by a value of $1 / e$.

As mentioned before, when the antenna is illuminated by an incident plane wave, axially symmetrical SPP modes are excited on the antenna. In particular, for the design of an efficient antenna, we are interested in the propagation properties of Transverse Magnetic (TM) SPP wave modes [3]. To obtain $k_{s p p}$, we need to solve the dispersion equation for SPP waves on the nano-antenna arms. We assume that the electric field $\vec{E}$ and magnetic field $\vec{H}$ on the antenna have the following form [7]:

$$
\begin{aligned}
& E_{r}(r, z)=E_{r}(r) e^{j k_{s p p} z}, \\
& E_{z}(r, z)=E_{z}(r) e^{j k_{s p p} z}, \\
& H_{\phi}(r, z)=H_{\phi}(r) e^{j k_{s p p} z} .
\end{aligned}
$$

Starting from the Maxwell's equations in their differential form and taking into account that for an axially symmetrical mode $\frac{\partial E}{\partial \phi}=0$ and $\frac{\partial H}{\partial \phi}=0$, the following equations can be written:

$$
\begin{aligned}
E_{r}(r, z) & =\frac{-j}{\varepsilon k_{0}} \frac{\partial H_{\phi}(r, z)}{\partial z}, \\
E_{z}(r, z) & =\frac{j}{\varepsilon k_{0}} \frac{1}{r} \frac{\partial\left(r H_{\phi}(r, z)\right)}{\partial r}, \\
H_{\phi}(r, z) & =\frac{-j}{\mu_{0} k_{0}}\left(\frac{\partial E_{r}(r, z)}{\partial z}-\frac{\partial E_{z}(r, z)}{\partial r}\right),
\end{aligned}
$$

where $\varepsilon=\varepsilon_{m}$ is the relative permittivity of the region $z<h$ or $r<a$ and $\varepsilon=\varepsilon_{d}$ is the relative permittivity of the region $z>h$ or $r>a, k_{0}=\omega / c$ refers to free-space wave vector and $c$ is the speed of light. By taking the $z$ derivative of (7), the radial component of the electric field can be written as:

$$
E_{r}(r, z)=\varepsilon^{-1} \frac{k_{s p p}}{k_{0}} H_{\phi}(r, z)
$$

By substituting (11) into (10) and taking again the $z$ derivative of (7), the magnetic field can be written as:

$$
H_{\phi}(r, z)=\frac{-j k_{0} \varepsilon}{k_{s p p}^{2}-k_{0}^{2} \varepsilon} \frac{d E_{z}(r, z)}{d r} .
$$


Finally, by substituting (12) into (9), the following equation for $E_{z}$ is obtained:

$$
\zeta^{2} \frac{d^{2} E_{z}(r, z)}{d \zeta^{2}}+\zeta \frac{d E_{z}(r, z)}{d \zeta}-\zeta^{2} E_{z}(r, z)=0,
$$

where $\zeta=p_{m, d} r$ and $p_{m, d}=\sqrt{k_{s p p}^{2}-k_{0}^{2} \varepsilon_{m, d} \mu_{0}}$ [7]. The solution of (13) for $E_{z}(r, z)$ has the form of $I_{0}\left(p_{m} a\right)$ in the metal and $K_{0}\left(p_{d} a\right)$ in the surrounding dielectric, where $I_{n}($. and $K_{n}($.$) are the generalized Bessel functions of first and$ second kind of order $n$. Substituting them into (12), and using the relations $\frac{d I_{0}\left(p_{m} r\right)}{d r}=p_{m} I_{1}\left(p_{m} r\right)$ and $\frac{d K_{0}\left(p_{d} r\right)}{d r}=$ $-p_{d} K_{1}\left(p_{d} r\right)$, the solution of $H_{\phi}(r, z)$ can be determined. In particular, by taking into account the need for continuity in $E_{z}(r, z)$ and $H_{\phi}(r, z)$ at the surface of the antenna, i.e., at the interface between the surrounding dielectric and the metal, $r=a$, the following dispersion equation is obtained:

$$
\frac{K_{0}\left(p_{d} a\right) I_{1}\left(p_{m} a\right)}{K_{1}\left(p_{d} a\right) I_{0}\left(p_{m} a\right)}=-\frac{\varepsilon_{d} p_{m}}{\varepsilon_{m} p_{d}}
$$

which can only numerically be solved. Ultimately, $k_{s p p}$ depends on the angular frequency $\omega$, the cylinder radius $a$ and the relative permittivities $\varepsilon_{m, d}$ of the metal and the surrounding dielectric. In Figure 3(a), the confinement factor (3) for gold-based nano-antennas is shown as a function of frequency and for different nanowire radius. The confinement factor increases as the frequency increases. It can also be seen that increasing the radius leads to a slow increase with frequency and, oppositely, the thiner the wire, the faster the confinement factor increases with frequency. Similarly, in Figure 3(b), the SPP wave propagation length (4) is shown as a function of the frequency. Ultimately, the smaller the radius, the higher the confinement factor is but the shorter the SPP propagation length becomes.

\subsection{Effective Resonance Length}

Besides the boundary condition imposed in the radial direction, there is another boundary effect that alters the propagation of SPP waves on the nano-antenna: the caps at the two ends. SPP waves travel along the wire length $l$ with a propagation constant $k_{s p p}$ and suffer a reflection at the two caps. To incorporate such effect in the analysis, the reflection coefficient $\mathcal{R}$ at the cap needs to be computed.

Following the methodology introduced in [11], by taking into account the boundary condition at the cap, we can write:

$$
\begin{aligned}
& E_{r}\left(r, z=h^{-}\right)=(1+\mathcal{R}) \frac{\operatorname{Re}\left\{k_{s p p}\right\}}{\omega \varepsilon_{0} \varepsilon} F(r), \\
& H_{\phi}\left(r, z=h^{-}\right)=(1-\mathcal{R}) F(r),
\end{aligned}
$$

with

$$
F(r)= \begin{cases}\frac{I_{1}\left(p_{m} r\right)}{I_{1}\left(p_{m} a\right)}, \varepsilon=\varepsilon_{m} & \text { if } r<a, \\ \frac{K_{1}\left(p_{d} r\right)}{K_{1}\left(p_{d} a\right)}, \varepsilon=\varepsilon_{d} & \text { if } r>a,\end{cases}
$$

and

$$
\begin{aligned}
& E_{r}\left(r, z=h^{+}\right)=\int_{0}^{+\infty} t(\eta) \frac{\sqrt{k_{0}^{2} \varepsilon_{d}-\eta^{2}}}{\omega \varepsilon_{d} \varepsilon_{0}} J_{1}(\eta \mathcal{R}) d \eta \\
& H_{\phi}\left(r, z=h^{+}\right)=\int_{0}^{+\infty} t(\eta) J_{1}(\eta \mathcal{R}) d \eta
\end{aligned}
$$

where $J_{1}($.$) is the first order Bessel function of the first$ kind. To solve the reflection coefficient $\mathcal{R}$, first we should

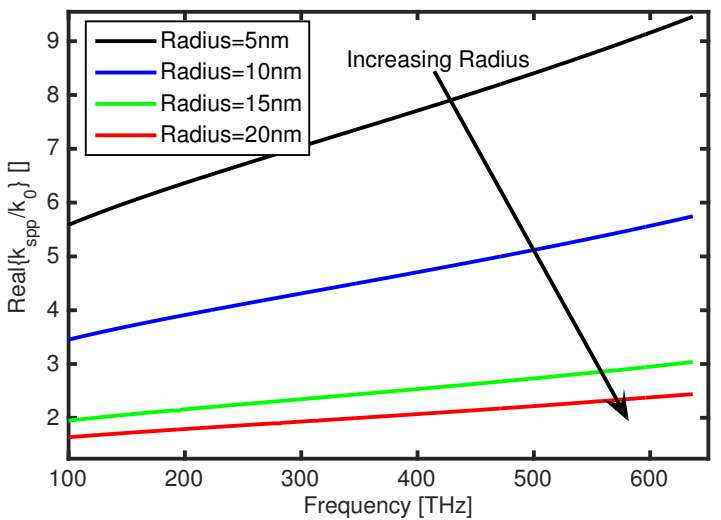

(a) Confinement Factor

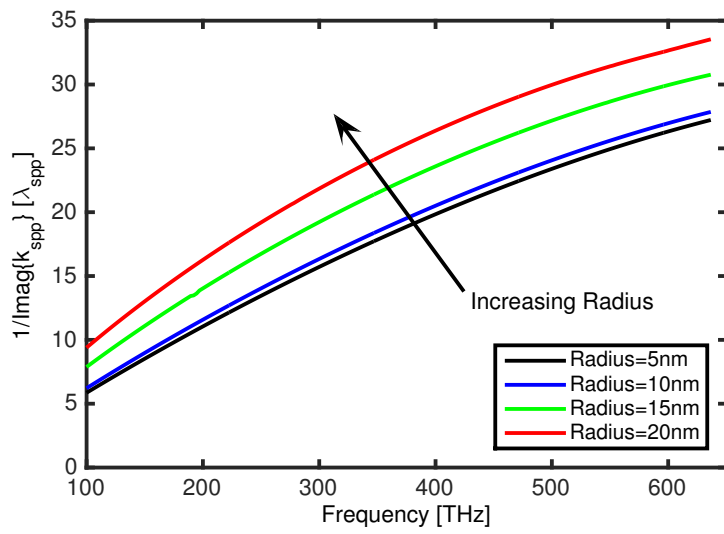

(b) Propagation Length

Figure 3: Propagation properties of SPP TM modes on gold nanowires.

find $t$. Hence by using the orthogonality property of Bessel functions of the first kind, (18) is equated to (15), both sides multiplied by $J_{1}(\eta r) r$ and then integrated from 0 to $\infty$ over $r$ to have:

$$
t(\eta)=(1+\mathcal{R}) \frac{\eta a \varepsilon_{d} \operatorname{Re}\left\{k_{s p p}\right\}}{\varepsilon_{0} \omega \sqrt{k_{0}^{2} \varepsilon_{d}-\eta^{2}}}\left[A_{1}(\eta)+A_{2}(\eta)\right],
$$

where

$$
A_{1}(\eta)=\frac{p_{m} I_{2}\left(p_{m} a\right) J_{1}(\eta a)+\eta I_{1}\left(p_{m} a\right) J_{2}(\eta a)}{I_{1}\left(p_{m} a\right) \varepsilon_{m}\left(\eta^{2}+p_{m}^{2}\right)}
$$

and

$$
A_{2}(\eta)=\frac{p_{d} K_{2}\left(p_{d} a\right) J_{1}(\eta a)-\eta K_{1}\left(p_{d} a\right) J_{2}(\eta a)}{K_{1}\left(p_{d} a\right) \varepsilon_{d}\left(\eta^{2}+p_{d}^{2}\right)} .
$$

For the magnetic field $H_{\phi},(19)$ is equated to (16). By multiplying both sides by $\frac{F(r) r}{\varepsilon}$ and following the same rule for $\varepsilon$ depending on $z$ and $r$, then integrating from 0 to $\infty$ over $r$, the final result would be:

$$
\mathcal{R}=\frac{1-G}{1+G}
$$




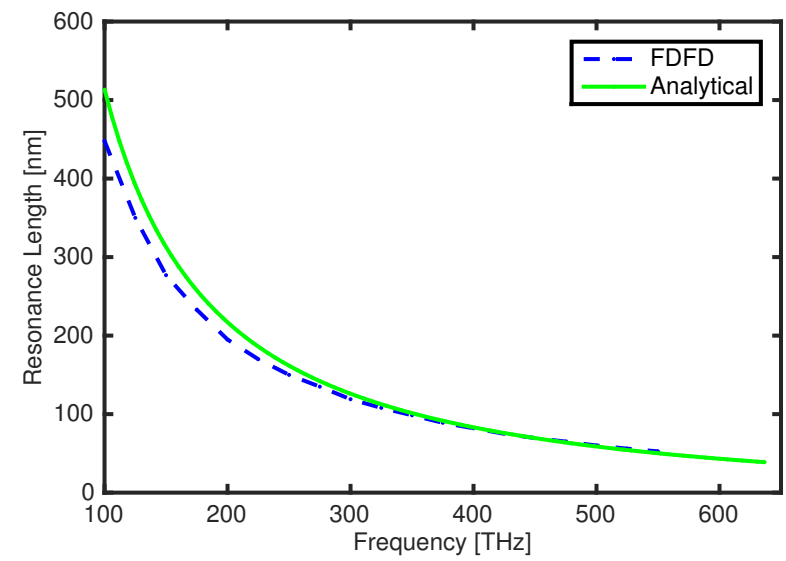

Figure 4: Resonance length of a gold-based plasmonic nano-antenna as a function of frequency (radius $a=5 \mathbf{n m}$ ).

where $\mathrm{G}$ is given by:

$$
G=\frac{\int_{0}^{+\infty} \frac{2 \eta \varepsilon_{d} \operatorname{Re}\left\{k_{s p p}\right\}}{\sqrt{k_{0}^{2} \varepsilon_{d}-\eta^{2}}}\left[A_{1}(\eta)+A_{2}(\eta)\right]^{2} d \eta}{\frac{I_{1}\left(p_{m} a\right)^{2}-I_{0}\left(p_{m} a\right) I_{2}\left(p_{m} a\right)}{\varepsilon_{m} I_{1}\left(p_{m} a\right)^{2}}-\frac{K_{1}\left(p_{d} a\right)^{2}-K_{0}\left(p_{d} a\right) K_{2}\left(p_{d} a\right)}{\varepsilon_{d} K_{1}\left(p_{d} a\right)^{2}}} .
$$

Finally, the resonances of the nanowire can be calculated by considering the propagation of the surface plasmon along the nanowire and reflection at two caps as [11]:

$$
l=\frac{n \pi-\varphi}{\operatorname{Re}\left\{k_{s p p}\right\}},
$$

where $n$ is the resonance order, $\varphi$ is the phase of the reflection coefficient $\mathcal{R}$ calculated from (23), and $k_{s p p}$ is the wave vector calculated by (14). As before, this can only be numerically solved. In Figure 4, the resonance length for gold-based nano-antennas is shown as a function of frequency. The length of a resonace optical nano-antenna is shorter than what an ideal PEC antenna would require to resonate at the same frequency, due to the large confinement factor of SPP waves in gold nanowires. In the same figure, the numerical results obtained by solving (24) are compared with that of running Finite-Differences Frequency-Domain (FDFD) simulations with COMSOL Multi-physics, for validation purposes.

\section{PLASMONIC NANO-ANTENNA PERFORMANCE IN RECEPTION}

In reception, we are interested in characterizing the plasmonic current generated at the nano-antenna as a result of the incident EM wave. In order to determine the plasmonic current, we follow the conventional vector potential approach [3], but contrary to classical antenna theory for PEC antennas, we take into account that there is an electric field inside the antenna, within the penetration depth, which is the SPP wave that propagates with $k_{\text {spp }}$.

At the nanowire surface, the following boundary condition between the plasmonic field inside the nano-antenna, the incident field on the nano-antenna and the scattered field by the nano-antenna needs to be satisfied:

$$
E_{z}^{\text {Inside }}(r, z)=E_{z}^{I n c}(r, z)+E_{z}^{S c a t}(r, z) .
$$

To simplify our analysis, we make the assumption that the nanowire is effectively very thin and, thus, the current density $J_{z}$, the vector potential $A_{z}$ and the electric field inside the antenna $E_{z}^{\text {Inside }}$ depend only on $z$ but not on $r$, i.e.,

$$
\begin{aligned}
J_{z}(r, z) & \approx J_{z}(z), \\
A_{z}(r, z) & \approx A_{z}(z), \\
E_{z}^{\text {Inside }}(r, z) & \approx E_{z}^{\text {Inside }}(z) .
\end{aligned}
$$

The field inside the nano-antenna $E_{z}^{i n c}$ in (26) is related to the current density through the material conductivity, i.e.,

$$
J_{z}(z)=\sigma(\omega) E_{z}^{\text {Inside }}(z)
$$

where $\sigma$ represents conductivity given by (2).

The incident field $E_{z}^{I n c}$ in (26) on the nano-antenna surface is given by (1).

From [10], the scattered field by the nano-antenna, $E_{z}^{\text {Scat }}$ in (26), can be written as

$$
E_{z}^{S c a t}(a, z)=\frac{j c^{2}}{\omega}\left(\frac{\partial^{2}}{\partial z^{2}}+k_{0}^{2}\right) A_{z}(a, z),
$$

where

$$
A_{z}(z) \approx \frac{\mu_{0}}{4 \pi} \bar{Z} J_{z}(z),
$$

and $\bar{Z}$ is the characteristic impedance of the wire, given by

$$
\bar{Z}=\frac{4 \pi}{\left(\varepsilon_{m}-1\right)\left(k_{s p p}^{2}-k_{0}^{2}\right)},
$$

where $\varepsilon_{m}$ is the metal permittivity given in (2) and $k_{s p p}$ is the SPP wave vector obtained from (14).

By combining (30), (31), (32), (33), and (1) in (26), the following inhomogeneous differential equation is obtained:

$$
E_{\|}^{I n c} e^{j k_{\|} z}+\frac{j c^{2}}{\omega}\left(\frac{\partial^{2}}{\partial z^{2}}+k_{0}^{2}\right) \frac{\mu_{0}}{4 \pi} \bar{Z} J_{z}(z)=\frac{1}{\sigma(\omega)} J_{z}(z) .
$$

Then, as analytically shown in [10], the total current in the nano-antenna $J_{z}$ can be written as the combination of the following terms

$$
J_{z}(z)=J_{\| \mid} e^{j k_{\| \mid} z}+J_{ \pm p} e^{ \pm j k_{s p p} z},
$$

where $J_{\|} e^{i k_{\| \mid} z}$ is induced by the illuminating field along the wire and $J_{ \pm p}$ refers to two counter-propagating plasmonic current densities propagating with propagation constant $k_{\text {spp }}$.

By combining (35) and (34), an expression for $J_{\| \text {can be }}$ found:

$$
J_{\|}=\sigma(\omega) \frac{\left(k_{s p p}^{2}-k_{0}^{2}\right)}{k_{s p p}^{2}-k_{\|}^{2}} E_{\|}^{I n c} .
$$

By considering the role of the wire end caps as a hard boundary and the vanishing total current density, an expression for $J_{ \pm p}$ is obtained as:

$$
J_{ \pm p}=-J_{\|} \frac{\sin \left(\left(k_{s p p} \pm k_{||}\right) h\right)}{\sin \left(2 k_{s p p} h\right)},
$$

where $h=\frac{l}{2}$ and $l$ stands for the length of wire given by (25). Finally, by combining (37) and (36), the plasmonic current density can be obtained as a function of the incident field $E_{z}^{i n c}$. As before, this can only be numerically done. 


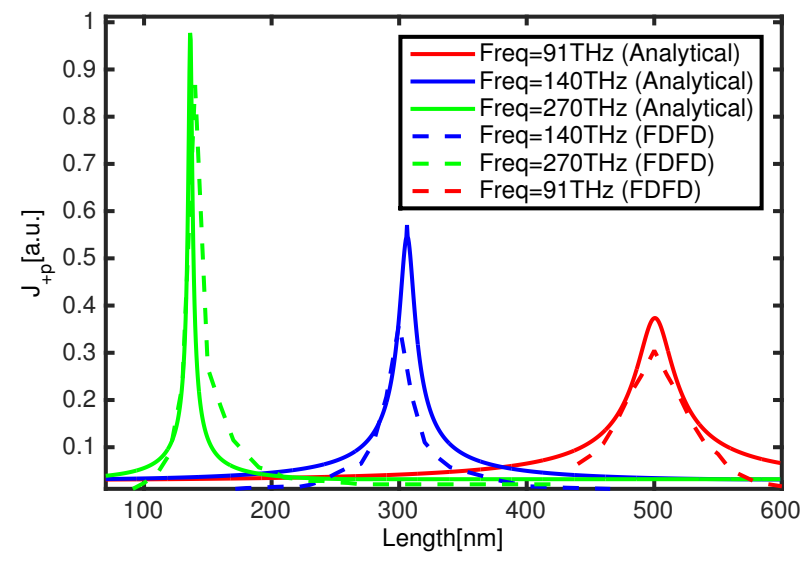

(a) $J_{+p}$ vs nano-antenna length

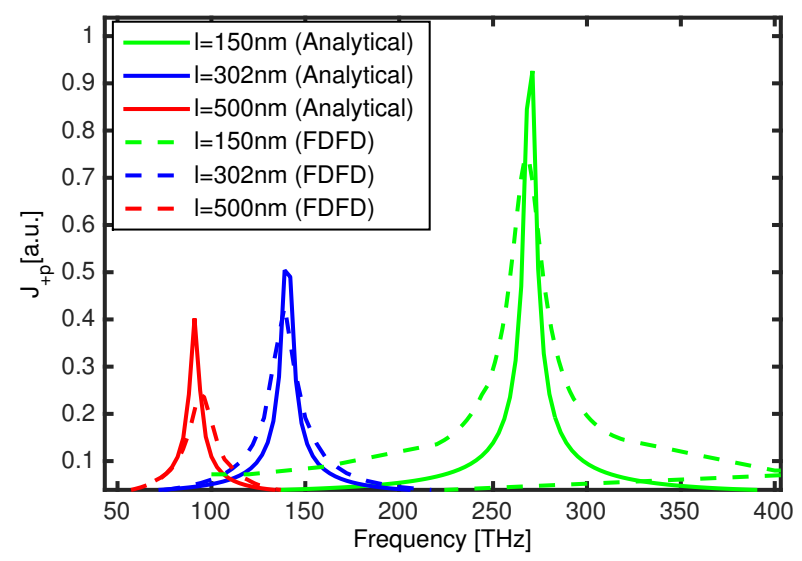

(b) $J_{+p}$ vs frequency

Figure 5: Plasmonic current density on a gold-based nano-antenna as a function of frequency and length (radius $a=5 \mathbf{n m}$ ).

\section{NUMERICAL AND SIMULATION RESULTS}

In this section, we numerically investigate the behavior of optical nano-antennas in reception. In this section, we consider gold-based nano-antennas with $5 \mathrm{~nm}$ radius and varying length. On the one hand, we numerically solve the plasmonic current density $J_{ \pm p}$ given by (37), which depends on its turn on the SPP wave propagation constant $k_{s p p}$, resonant length $l$ and material conductivity $\sigma$ parameters given by (14), (25) and (2), respectively. On the other other hand, we utilize COMSOL Multi-physics to validate our results by means of FDFD simulations, in which the only required initial parameter is the material conductivity $\sigma$.

In Figure 5(a), the plasmonic current density is shown as a function of the nano-antenna length $l$ for three different illumination frequencies, namely, $91 \mathrm{THz}, 140 \mathrm{THz}$ and $270 \mathrm{THz}$. Clear resonances can be observed when the length is approximately $500 \mathrm{~nm}, 302 \mathrm{~nm}$ and $150 \mathrm{~nm}$, respectively. The z-component of the current density and its maximum is taken from analytically produced data and FDTD sim- ulations for different length and frequencies are performed for validation purposes. There is good agreement between the numerical and the simulation results. As expected, the high confinement factor of SPP waves in gold results into significantly smaller nano-antennas when compared to ideal PEC antennas at the same frequency.

Similarly, in Figure 5(b), the plasmonic current is shown as a function of the frequency of the incident EM field, for different nano-antenna lengths. The longest dipole $(500 \mathrm{~nm})$ exhibits the weakest response while the shortest nanowire (140 nm) exhibits the strongest response. Multiple resonances can be observed for each nano-wire length, but we are mainly interested in the fundamental resonance frequency.

\section{CONCLUSIONS AND FUTURE WORK}

In this paper, we have developed a unified mathematical framework to analyze the performance in reception of plasmonic nano-antennas in reception. Starting from the dynamic complex conductivity of the nano-antenna building components, we have formulated the dispersion equation for SPP waves in nanowires and obtained the SPP wave propagation properties and nano-antenna fundamental resonance length by taking into account also the impact of the nanowire caps. We have then analytically derived an expression for the plasmonic current in the nano-antenna when irradiated by an incident EM wave. The analytical models have been then validated by means of FDFD simulations with COMSOL Multi-physics. The developed framework can be utilized to guide the design and development of novel nano-antennas suited for wireless optical communication in intra-body nanonetworks. As part of our future work, we will extend our analysis to the nano-antenna in transmission, and we will also compare the nano-antenna performance, both in transmission and in reception, when utilizing different materials.

\section{ACKNOWLEDGMENTS}

This work was supported by the U.S. National Science Foundation (NSF) under Grant No. CBET-1445934.

\section{REFERENCES}

[1] I. F. Akyildiz and J. M. Jornet. Electromagnetic wireless nanosensor networks. Nano Communication Networks (Elsevier) Journal, 1(1):3-19, Mar. 2010.

[2] I. F. Akyildiz, M. Pierobon, S. Balasubramaniam, and Y. Koucheryavy. The internet of bio-nano things. IEEE Communications Magazine, 53(3):32-40, 2015.

[3] C. A. Balanis. Antenna theory: analysis and design. John Wiley \& Sons, 2012.

[4] P. Biagioni, J.-S. Huang, and B. Hecht. Nanoantennas for visible and infrared radiation. Reports on Progress in Physics, 75(2):024402, 2012.

[5] A. Boltasseva and H. A. Atwater. Low-loss plasmonic metamaterials. Science, 331(6015):290-291, 2011.

[6] A. G. Brolo. Plasmonics for future biosensors. Nature Photonics, 6(11):709-713, 2012.

[7] Q. Cao and J. Jahns. Azimuthally polarized surface plasmons as effective terahertz waveguides. Optics express, 13(2):511-518, 2005.

[8] I. Choi and Y. Choi. Plasmonic nanosensors: Review and prospect. IEEE Journal of Selected Topics in Quantum Electronics, 18(3):1110-1121, 2012. 
[9] J. Dorfmüller. Optical Wire Antennas: Near-Field Imaging, Modeling and Emission Patterns. PhD thesis, Citeseer, 2010.

[10] J. Dorfm§ller, R. Vogelgesang, R. T. Weitz, C. Rockstuhl, C. Etrich, T. Pertsch, F. Lederer, and K. Kern. Fabry-pérot resonances in one-dimensional plasmonic nanostructures. Nano letters, 9(6):2372-2377, 2009.

[11] R. Gordon. Reflection of cylindrical surface waves. Optics express, 17(21):18621-18629, 2009.

[12] S. L. Jacques. Optical properties of biological tissues: a review. Physics in medicine and biology, 58(11):R37, 2013.

[13] P. B. Johnson and R.-W. Christy. Optical constants of the noble metals. Physical Review B, 6(12):4370, 1972.

[14] J. M. Jornet and I. F. Akyildiz. Channel modeling and capacity analysis of electromagnetic wireless nanonetworks in the terahertz band. IEEE Transactions on Wireless Communications, 10(10):3211-3221, Oct. 2011.

[15] J. M. Jornet and I. F. Akyildiz. Graphene-based plasmonic nano-antenna for terahertz band communication in nanonetworks. Selected Areas in Communications, IEEE Journal on, 31(12):685-694, 2013.

[16] G. Kristensson, S. Rikte, and A. Sihvola. Mixing formulas in the time domain. JOSA A, 15(5):1411-1422, 1998.

[17] A. Mohammadi, V. Sandoghdar, and M. Agio. Gold, copper, silver and aluminum nanoantennas to enhance spontaneous emission. Journal of Computational and Theoretical Nanoscience, 6(9):2024-2030, 2009.

[18] Q.-H. Park. Optical antennas and plasmonics. Contemporary physics, 50(2):407-423, 2009.

[19] G. Piro, K. Yang, G. Boggia, N. Chopra, L. Grieco, and A. Alomainy. Terahertz communications in human tissues at the nano-scale for healthcare applications. IEEE Transactions on Nanotechnology, 14(3):404-406, 2015.

[20] G. E. Santagati, T. Melodia, L. Galluccio, and S. Palazzo. Ultrasonic networking for e-health applications. IEEE Wireless Communications, 20(4), 2013.

[21] T. Stauber, N. M. R. Peres, and A. K. Geim. Optical conductivity of graphene in the visible region of the spectrum. Physical Review B, 78:085432, Aug. 2008.

[22] A. Vakil and N. Engheta. Transformation optics using graphene. Science, 332(6035):1291-1294, 2011.

[23] P. R. West, S. Ishii, G. V. Naik, N. K. Emani, V. M. Shalaev, and A. Boltasseva. Searching for better plasmonic materials. Laser 83 Photonics Reviews, 4(6):795-808, 2010. 